\title{
$D$ meson semileptonic decays in lattice QCD with Möbius domain-wall quarks
}

\author{
JLQCD Collaboration: T. Kaneko ${ }^{\star a, b}$, B. Fahy ${ }^{a}$, H. Fukaya ${ }^{c}$, S. Hashimoto ${ }^{a, b}$ \\ ${ }^{a}$ High Energy Accelerator Research Organization (KEK), Ibaraki 305-0801, Japan \\ ${ }^{b}$ School of High Energy Accelerator Science, SOKENDAI (The Graduate University for \\ Advanced Studies), Ibaraki 305-0801, Japan \\ ${ }^{c}$ Department of Physics, Osaka University, Osaka 560-0043, Japan \\ E-mail: takashi.kaneko@kek.jp
}

\begin{abstract}
We report on our study of the $D$ meson semileptonic decays in $2+1$ flavor lattice QCD. Gauge ensembles are generated at three lattice cutoffs up to $4.5 \mathrm{GeV}$ and with pion masses as low as $300 \mathrm{MeV}$. We employ the Möbius domain-wall fermion action for both light and charm quarks. We report our preliminary results for the vector and scalar form factors and discuss their dependence on the momentum transfer, quark masses and lattice spacing.
\end{abstract}

34th annual International Symposium on Lattice Field Theory

24-30 July 2016

University of Southampton, UK

\footnotetext{
* Speaker.
} 


\section{Introduction}

The $D \rightarrow \pi l v$ and $D \rightarrow K l v$ semileptonic decays provide a precise determination of the CabibboKobayashi-Maskawa matrix elements $\left|V_{c d}\right|$ and $\left|V_{c s}\right|$, and play an important role in the search for new physics in the charm sector [1]. The vector and scalar form factors $f_{\{+, 0\}}^{D P}$ describe nonperturbative QCD effects, and are defined from the relevant hadronic matrix elements as

$$
\begin{aligned}
\left\langle P\left(p^{\prime}\right)\left|V_{\mu}\right| D(p)\right\rangle & =\left(p+p^{\prime}\right)_{\mu} f_{+}^{D P}(t)+\left(p-p^{\prime}\right)_{\mu} f_{-}^{D P}(t), \\
f_{0}^{D P}(t) & =f_{+}^{D P}(t)+\frac{t}{M_{D}^{2}-M_{P}^{2}} f_{-}^{D P}(t)
\end{aligned}
$$

where $P$ specifies the light meson $(P=\pi, K)$ and $t=\left(p-p^{\prime}\right)^{2}$ is the momentum transfer. The current accuracy of $\left|V_{c d}\right|$ and $\left|V_{c s}\right|$ is limited by the theoretical uncertainty of $f_{\{+, 0\}}^{D P}$ [2]. Lattice QCD is the only known method to calculate $f_{\{+, 0\}}^{D P}$ with controlled and systematically-improvable uncertainties.

In this article, we report on our calculation of these form factors in $N_{f}=2+1 \mathrm{QCD}$ with the tree-level improved Symanzik gauge action and the Möbius domain wall quark action [3]. Numerical simulations are carried out at three lattice cutoffs $a^{-1} \sim 2.5,3.6$ and $4.5 \mathrm{GeV}$. On such fine lattices, we employ the domain-wall action also for charm quarks. The simulated values of $m_{u d}$, that is the mass of the degenerate up and down quarks, cover a range of the pion mass $300 \mathrm{MeV} \lesssim M_{\pi} \lesssim 500 \mathrm{MeV}$. We take a strange quark mass $m_{s}$ close to its physical value. An additional value of $m_{s}$ is simulated at certain choices of $\left(a, m_{u d}\right)$ in order to study the $m_{s}$ dependence of the form factors. The charm quark mass is set to its physical value determined from the $D$ meson spectrum [4]. The physical charm quark mass extracted from the same set of simulations is $m_{c}(3 \mathrm{GeV})=1.003(10) \mathrm{GeV}$, which is consistent with the present world average [5]. Our simulation parameters are summarized in Table 1. After the previous report [6], we extend our simulation to the two finer lattices and improve our measurement method to reduce the statistical uncertainty.

At each simulation point, our lattice size satisfies a condition $M_{\pi} L \gtrsim 4$ to control finite volume effects, and we accumulate 5,000 Molecular Dynamics time. Chiral symmetry is preserved to good accuracy by choosing the sign function approximation and the kernel operator in the 4-dimensional

Table 1: Simulation parameters.

\begin{tabular}{l|llll|l}
\hline lattice parameters & $m_{u d}$ & $m_{s}$ & $M_{\pi}[\mathrm{MeV}]$ & $M_{K}[\mathrm{MeV}]$ & $N_{x_{4, \mathrm{src}}}$ \\
\hline$\beta=4.17, a^{-1}=2.453(4), 32^{3} \times 64 \times 12$ & 0.0190 & 0.0400 & $499(1)$ & $618(1)$ & 2 \\
& 0.0120 & 0.0400 & $399(1)$ & $577(1)$ & 2 \\
& 0.0070 & 0.0400 & $309(1)$ & $547(1)$ & 4 \\
\cline { 2 - 5 } & 0.0190 & 0.0300 & $498(1)$ & $563(1)$ & 2 \\
\hline$\beta=4.35, a^{-1}=3.610(9), 48^{3} \times 96 \times 8$ & 0.0120 & 0.0250 & $501(2)$ & $620(2)$ & 2 \\
& 0.0080 & 0.0250 & $408(2)$ & $582(2)$ & 2 \\
& 0.0042 & 0.0250 & $300(1)$ & $547(2)$ & 4 \\
\cline { 2 - 6 } & 0.0120 & 0.0180 & $499(1)$ & $557(2)$ & 2 \\
\hline$\beta=4.47, a^{-1}=4.496(9), 64^{3} \times 128 \times 8$ & 0.0030 & 0.0150 & $284(1)$ & $486(1)$ & 1 \\
\hline
\end{tabular}


effective action [7]. The residual mass is suppressed to $O(1 \mathrm{MeV})$ at the coarsest lattice, and even smaller $\lesssim 0.2 \mathrm{MeV}$ at finer lattices with moderate sizes in the fifth dimension $\sim 10$.

\section{Calculation of form factors}

We calculate the three-point function

$$
\begin{aligned}
C_{V_{\mu}}^{D P}\left(\mathbf{p}, \mathbf{p}^{\prime} ; \Delta x_{4}, \Delta x_{4}^{\prime}\right)= & \frac{1}{N_{s}^{3} N_{x_{4}, \mathrm{src}}} \sum_{x_{4, \mathrm{scc}}} \sum_{\mathbf{x}, \mathbf{x}^{\prime}, \mathbf{x}^{\prime \prime}}\left\langle\mathscr{O}_{P}\left(\mathbf{x}^{\prime \prime}, x_{4, \mathrm{src}}+\Delta x_{4}+\Delta x_{4}^{\prime}\right)\right. \\
& \left.\quad \times V_{\mu}\left(\mathbf{x}^{\prime}, x_{4, \mathrm{src}}+\Delta x_{4}\right) \mathscr{O}_{D}^{\dagger}\left(\mathbf{x}, x_{4, \mathrm{src}}\right)\right\rangle e^{-i \mathbf{p}^{\prime}\left(\mathbf{x}^{\prime \prime}-\mathbf{x}^{\prime}\right)} e^{-i \mathbf{p}\left(\mathbf{x}^{\prime}-\mathbf{x}\right),}
\end{aligned}
$$

where $N_{s}$ is the spatial lattice size and $\mathbf{p}^{(\prime)}$ represents the momentum of the initial (final) meson. We apply a Gaussian smearing to the meson interpolating operators $\mathscr{O}_{\{\pi, K, D\}}$. The temporal coordinate of the source operator is denoted by $x_{4, \text { src }}$, and $\Delta x_{4}{ }^{(\prime)}$ represents the temporal separation between the source (sink) operator and the vector current $V_{\mu}$.

In this study, we calculate $C_{V_{\mu}}^{D P}\left(\mathbf{p}, \mathbf{p}^{\prime} ; \Delta x_{4}, \Delta x_{4}^{\prime}\right)$ by varying $\Delta x_{4}$ with $\Delta x_{4}+\Delta x_{4}^{\prime}$ kept fixed. Its physical length is the same for the three cutoffs and is chosen as $\Delta x_{4}+\Delta x_{4}^{\prime}=28 a$ at $\beta=4.17$ [6]. The $D$ meson is at rest $(\mathbf{p}=\mathbf{0})$, and we simulate four different values of the momentum transfer $t$ with light meson momenta $\left|\mathbf{p}^{\prime}\right|^{2}=0,1,2,3$ in units of $(2 \pi / L)^{2}$. For $P=\pi(K)$, the minimum value of the momentum transfer is typically $t_{\min } \approx 0.3(0.2) \mathrm{GeV}^{2}$, while the maximum is $t_{\max } \approx 2.6(1.8) \mathrm{GeV}^{2}$.

We also calculate two-point functions of $\pi, K$ and $D$ mesons. The amplitudes of the correlators are extracted by the following exponential fits in terms of $\Delta x_{4}$

$$
\begin{aligned}
C_{V_{\mu}}^{D P}\left(\mathbf{p}, \mathbf{p}^{\prime} ; \Delta x_{4}, \Delta x_{4}^{\prime}\right) & =A_{V_{\mu}}^{D P}\left(\mathbf{p}, \mathbf{p}^{\prime}\right) e^{-E_{D}(\mathbf{p}) \Delta x_{4}} e^{-E_{P}\left(\mathbf{p}^{\prime}\right) \Delta x_{4}^{\prime}} \quad(P=\pi, K), \\
C^{Q}\left(\mathbf{p} ; \Delta x_{4}\right) & =B^{Q}(\mathbf{p}) e^{-E_{Q}(\mathbf{p}) \Delta x_{4}} \quad(Q=\pi, K, D) .
\end{aligned}
$$

Here the meson energies $E_{\{\pi, K, D\}}$ are estimated from their rest masses [4] and the dispersion relation in the continuum limit. The matrix elements are given as

$$
\left\langle P\left(\mathbf{p}^{\prime}\right)\left|V_{\mu}\right| D(\mathbf{p})\right\rangle=2 Z_{V} \sqrt{\frac{E_{D}(\mathbf{p}) E_{P}\left(\mathbf{p}^{\prime}\right)\left|A_{V_{\mu}}^{D P}\left(\mathbf{p}, \mathbf{p}^{\prime}\right)\right|^{2}}{B^{D}(\mathbf{p}) B^{P}\left(\mathbf{p}^{\prime}\right)}},
$$

where we use the renormalization factor $Z_{V}$ non-perturbatively calculated in Ref. [8]. The relevant semileptonic form factors are then extracted via Eqs. (1.1) and (1.2).

In order to improve the statistical accuracy, we average the three- and two-point functions over the locations of the source operator. As for the temporal location, we repeat our measurement over $N_{x_{4, \text { src }}}$ different values of $x_{4, \text { src }}$. Our choice of $N_{x_{4, \text { src }}}$ is summarized in Table 1. An important improvement from Ref. [6] is to average over the spatial coordinates $\mathbf{x}$ as well by putting the Gaussian source operator associated with a $Z_{2}$ noise at each lattice site at a given time-slice $x_{4, \text { src }}$.

Figure 1 compares our results for the amplitude $A_{V_{4}}^{D \pi}$ with different measurement setups on our coarsest lattice at the heaviest sea quark masses. We observe about $30 \%$ reduction of the statistical error by averaging over $2 t_{\text {src }}$ 's and an additional $30 \%$ reduction by averaging over $\mathbf{x}$ : about a factor of two improvement in total. Averaging over $\mathbf{p}$ further improves the statistical accuracy: at $|\mathbf{p}|^{2}=2$, for instance, about factor of two improvement by averaging over $12 \mathbf{p}$ 's. The typical statistical accuracy is $1-2 \%$ at $t_{\max }$ and $M_{\pi} \sim 500 \mathrm{MeV}$, and $6-9 \%$ at $t_{\min }$ and $M_{\pi} \sim 300 \mathrm{MeV}$. 


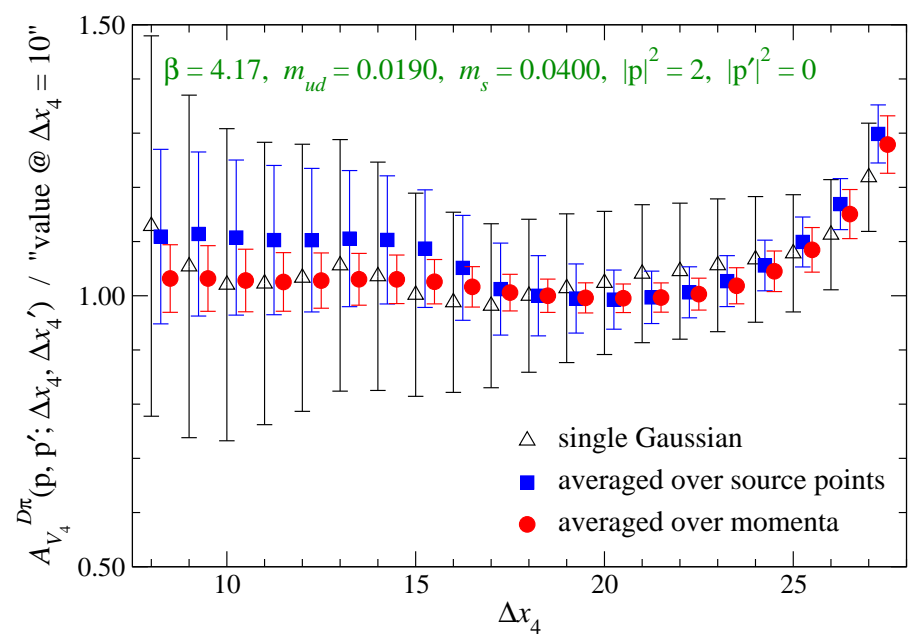

Figure 1: Effective value of amplitude $A_{V_{4}}^{D \pi}\left(\mathbf{p}, \mathbf{p}^{\prime}\right)$ as a function of $\Delta x_{4}$. We plot data with $|\mathbf{p}|=2$ and $\left|\mathbf{p}^{\prime}\right|^{2}=0$ at $\beta=4.17$ and $\left(m_{u d}, m_{s}\right)=(0.0190,0.0400)$. The open triangles show data with a "local" Gaussian source and a single choice of $\mathbf{p}$. The blue squares and red circles are obtained by averaging over the location of the source operator and then over 12 p's. All data are normalized by their value at $\Delta x_{4}=18$.

\section{Momentum transfer dependence}

We parametrize the momentum transfer dependence of the form factors in terms of the socalled $z$ parameter [9]

$$
z\left(t, t_{0}\right)=\frac{\sqrt{t_{+}-t}-\sqrt{t_{+}-t_{0}}}{\sqrt{t_{+}-t}+\sqrt{t_{+}-t_{0}}}
$$

where $t_{+}=\left(M_{D}+M_{P}\right)^{2}$ represents the $D P$ threshold $(P=\pi, K)$. The free parameter $t_{0}$ is chosen so that our simulated region $t \in\left[t_{\min }, t_{\max }\right]$ is mapped into a shortest segment $z \in\left[-|z|_{\max },+|z|_{\max }\right]$ centered at the origin. Typical size of the $z$ parameter is $|z|_{\max } \lesssim 0.2$.

The momentum transfer dependence of the form factors are then parametrized by using this small parameter as

$$
f_{\{+, 0\}}^{D P}(t)=\frac{1}{B(t)} \sum_{k=0}^{N_{\{+, 0\}}} a_{\{+, 0\}, k} z^{k} .
$$

In this preliminary analysis, we test two choices of the factor $B(t)$. In the so-called BourrelyCaprini-Lellouch (BCL) parametrization [10] with

$$
B(t)=1-\frac{t}{M_{\text {pole }}^{2}}
$$

possibly small deviation from the lowest pole contribution $1 / B(t)$ is expanded in terms of $z$. We also test a naive polynomial expansion of the form factors themselves with

$$
B(t)=1 .
$$




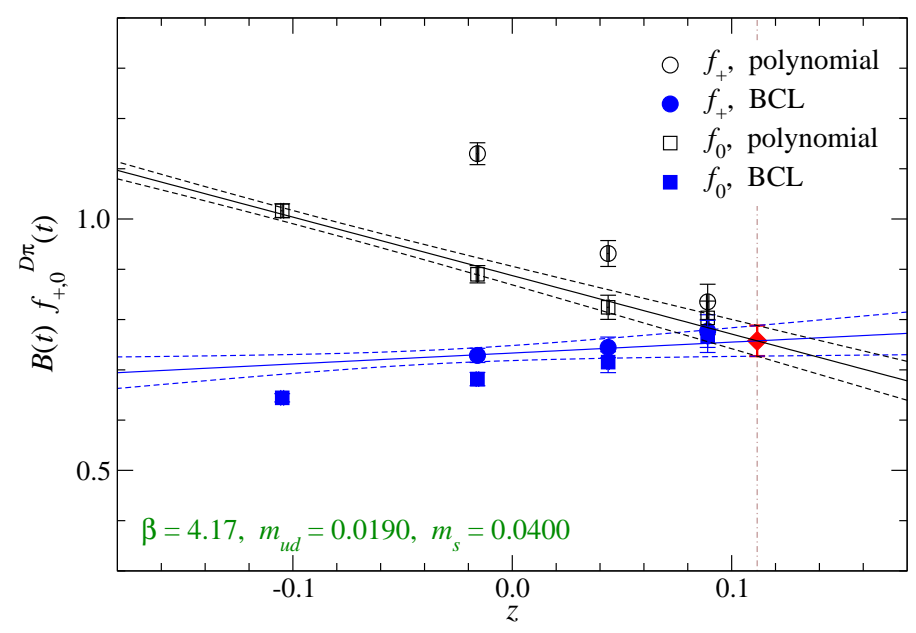

Figure 2: Plot of $B(t) f_{\{+, 0\}}^{D \pi}(t)$ as a function of $z$ at $\beta=4.17$ and $\left(m_{u d}, m_{s}\right)=(0.0190,0.0400)$. Circles and squares are data for $f_{+}$and $f_{0}$, respectively. Filled symbols show $\left(1-t / M_{D_{(0)}^{*}}^{2}\right) f_{+(0)}^{D \pi}(t)$ for the BCL parametrization, whereas open symbols for the polynomial expansion are just the form factors themselves. A simultaneous fit to $f_{+}$(filled symbols) and $f_{0}$ (open symbols) is shown by solid and dashed lines. The vertical dot-dashed line represents $z$ corresponding $t=0$, and the diamond is the value extrapolated to $t=0$.

Figure 2 shows $z$-dependence of a quantity $B(t) f_{\{+, 0\}}^{D \pi}(t)$ to be expanded in terms of $z$. Namely, $B(t) f_{+(0)}^{D \pi}(t)=\left(1-t / M_{D_{(0)}^{*}}^{2}\right) f_{+(0)}^{D \pi}(t)$ for the BCL parametrization with Eq. (3.3), whereas it is just the form factor for the polynomial expansion with Eq. (3.4).

For $f_{+}^{D P}$, we use the vector meson masses $M_{D_{(s)}^{*}}$ calculated at the simulation points, which are well below the threshold $t_{+}$. We observe that the $z$ dependence of $B(t) f_{+}^{D P}(t)$ is significantly reduced by switching from the polynomial expansion (3.4) to the BCL parametrization (3.3). This suggests that the vector meson dominance (VMD) hypothesis is a reasonably good approximation of $f_{+}^{D P}$, and we can expand the small deviation from the VMD in terms of small $z$. In this study, we test two BCL parametrization including the linear $\left(N_{+}=1\right)$ and quadratic terms $\left(N_{+}=2\right)$.

We have not yet calculated the scalar meson masses $M_{D_{(s)}^{*}}$, and hence it is not clear whether there exist corresponding isolated poles below $t_{+}$at simulated $M_{\pi}$ 's. In this analysis, we employ the simple linear expansion (3.4) for $f_{0}^{D P}$. We also test the BCL parametrization $\left(N_{0}=1\right)$ with the experimental value of $M_{D_{(s) 0}^{*}}$ by assuming its mild dependence on $m_{u d}$.

We estimate the normalization $f_{+}^{D P}(0)=f_{0}^{D P}(0)$ from the simultaneous fit using the BCL parametrization with $N_{+}=1$ for $f_{+}^{D P}$ and the linear parametrization $\left(N_{0}=1\right)$ for $f_{0}^{D P}$. We also test above-mentioned alternative forms to estimate the systematic uncertainty of the extrapolation to $t=0$. As shown in Fig. 2, however, the $z$ dependence of our results is mild except the polynomial parametrization for $f_{+}^{D P}$. The systematic uncertainty is not large compared to the statistical accuracy.

\section{Continuum and chiral extrapolation}

In Fig. 3, we compare $f_{\{+, 0\}}^{D \pi}$ at different pion masses (left panel) and at different lattice spac- 

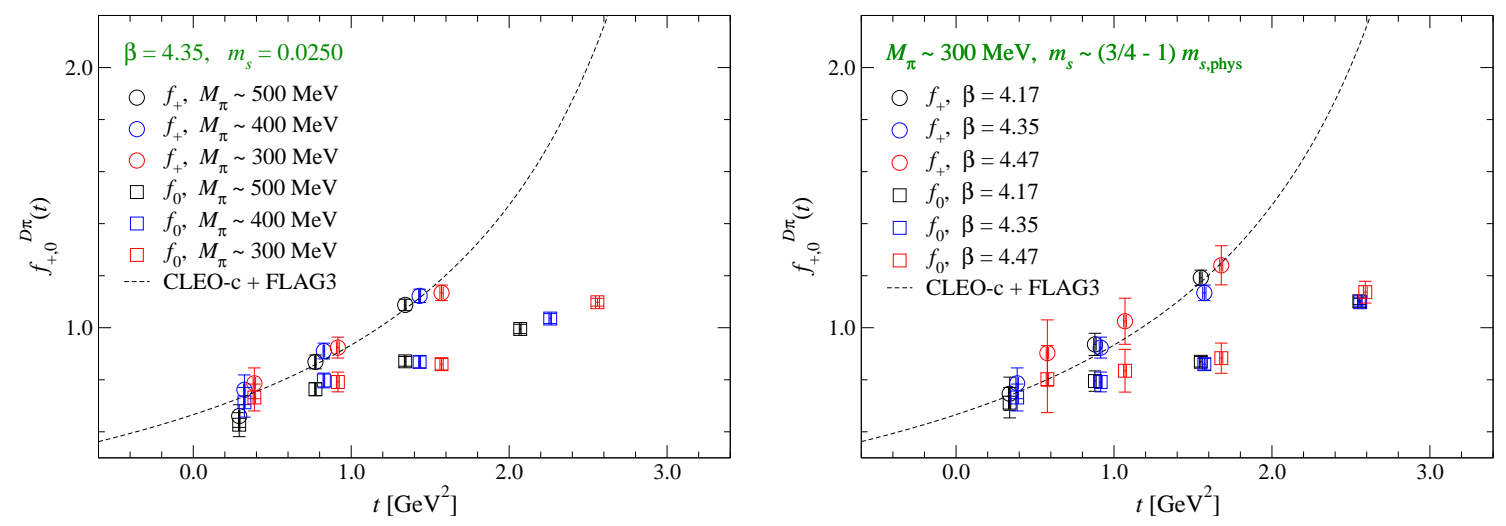

Figure 3: Comparison of $f_{\{+, 0\}}^{D \pi}$ among different $M_{\pi}$ 's (left panel) and different $a$ 's (right panel). We plot data at $\beta=4.35$ and $m_{s}=0.0250$ in the left panel, whereas the right panel shows data at $M_{\pi} \sim 300 \mathrm{MeV}$ and larger $m_{s}$. We also plot the Becirevic-Kaidalov parametrization [11] of the CLEO-c data of $f_{+}{ }^{D \pi}(t)$ [12] combined with an average of recent lattice estimates of $f_{+}{ }^{D \pi}(0)$ [13].

ings (right panel). The reasonable consistency in both panels suggests a mild dependence on $M_{\pi}$ and $a$. We note that the decay constants $f_{D_{(s)}}$ also have small discretization errors with our choice of the lattice action and cutoffs [14].

In this preliminary analysis, therefore, we parametrize the $a, m_{u d}$ and $m_{s}$ dependences of $f_{+}^{D P}(0)$ by the following simple linear form

$$
f_{+}^{D P}(0)=c^{D P}+c_{a}^{D P} a^{2}+c_{\pi}^{D P} M_{\pi}^{2}+c_{\eta_{s}}^{D P} M_{\eta_{s}}^{2},
$$

where $M_{\eta_{s}}^{2}=2 M_{K}^{2}-M_{\pi}^{2}$. This continuum and chiral extrapolation is plotted in Fig 4. We obtain $\chi^{2} /$ d.o.f $\sim 1.6-1.8$. All the coefficients $c_{\left\{a, \pi, \eta_{s}\right\}}^{D P}$ have $\gtrsim 75 \%$ statistical error: namely, consistent with zero as expected from the good consistency in Fig. 3. This fit is therefore not sensitive to higher order corrections, and we estimate the systematic uncertainty from three fits in which one of the three coefficients $c_{\left\{a, \pi, \eta_{s}\right\}}^{D P}$ is set to zero. Our preliminary estimates

$$
f_{+}^{D \pi}(0)=0.644(49)(27), \quad f_{+}^{D K}(0)=0.701(46)(33) .
$$

are consistent with recent lattice averages $f_{+}^{D \pi}(0)=0.666(29)$ and $f_{+}^{D K}(0)=0.747(19)$ [13].

\section{Summary}

In this article, we report on our lattice calculation of the $D \rightarrow \pi$ and $D \rightarrow K$ semileptonic form factors. We employ the Möbius domain-wall quark action both for light and charm quarks, and simulate lattice cutoffs up to $4.5 \mathrm{GeV}$.

Our preliminary results for $f_{+}{ }^{D \pi(K)}(0)$ have uncertainty of 8 (9) \%. We expect significant improvement in the near future by increasing statistics $\left(N_{x_{4, \mathrm{scc}}}\right)$ on the finest lattice and extending our measurements to smaller $M_{\pi} \sim 230 \mathrm{MeV}$.

We observe small discretization errors of the $D$ meson form factors with our simulation setup. It is therefore interesting to extend our study to the $B$ meson semileptonic decays, which are being precisely measured at SuperKEKB/Belle II and LHCb experiments. 

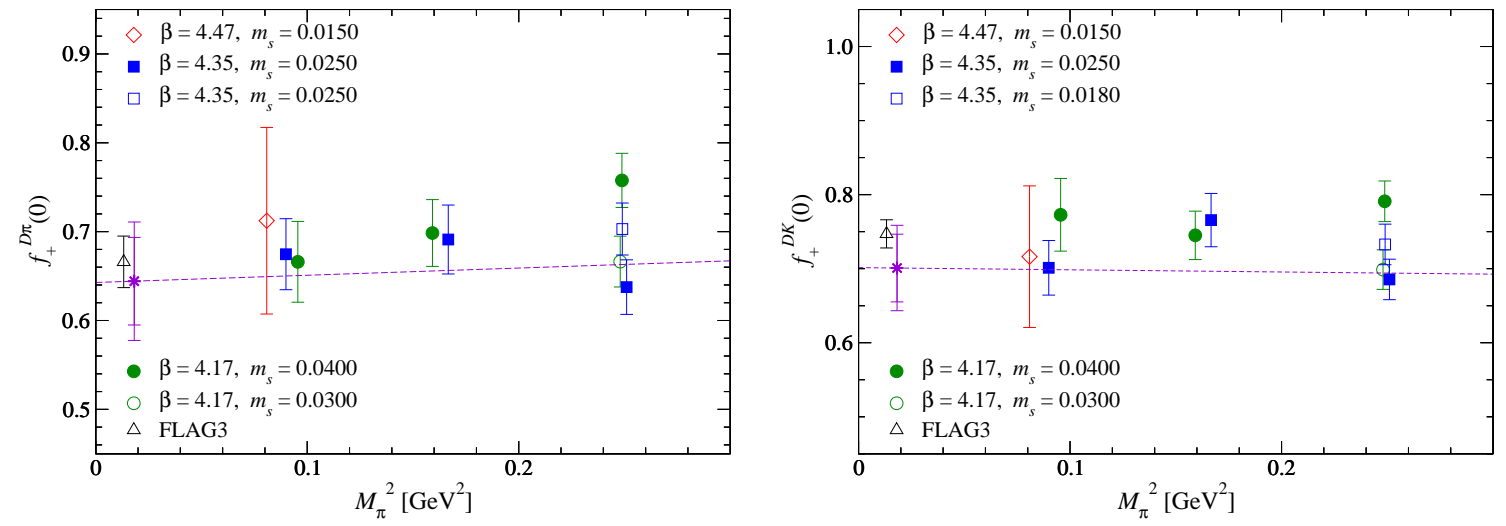

Figure 4: Continuum and chiral extrapolation of $f_{+}{ }^{D \pi}(0)$ (left panel) and $f_{+}{ }^{D K}(0)$ (right panel). Data at different $a$ 's and $m_{s}$ 's are plotted by different symbols as a function of $M_{\pi}^{2}$. The dashed lines show the fit line in the continuum limit and at the physical strange quark mass. The value extrapolated to the physical point is plotted by the stars. We also plot averages of recent lattice estimates [13] by the triangles.

Numerical simulations are performed on Hitachi SR16000 and IBM System Blue Gene Solution at KEK under a support of its Large Scale Simulation Program (No. 16/17-14). This research is supported in part by the Grant-in-Aid of the MEXT (No. 26247043, 26400259) and by MEXT as "Priority Issue on Post-K computer" (Elucidation of the Fundamental Laws and Evolution of the Universe) and JICFuS.

\section{References}

[1] J. Barranco et al., arXiv:1303.3896 [hep-ph].

[2] T. Kaneko, X.R. Lyu and A. Oyanguren, PoS (CKM2016) 014.

[3] R.C. Brower, H. Neff and K. Orginos, Nucl. Phys. (Proc.Suppl.) 140 (2005) 686.

[4] B. Fahy et al. (JLQCD Collaboration), PoS (LATTICE2015) 074.

[5] K. Nakayama, B. Fahy and S. Hashimoto (JLQCD Collaboration), Phys. Rev. D94 (2016) 054507.

[6] T. Suzuki et al. (JLQCD Collboration), PoS (LATTICE2015) 337.

[7] T. Kaneko et al. (JLQCD Collaboration), PoS (LATTICE 2013) 125.

[8] M. Tomii et al. (JLQCD Collaboration), Phys. Rev. D94 (2016) 054504.

[9] C. Bourrely, B. Machet and E. de Rafael, Nucl. Phys. B189 (1981) 157.

[10] C. Bourrely, I. Caprini and L. Lellouch, Phys. Rev. D79 (2009) 013008.

[11] D. Becirevic and A.B. Kaidalov, Phys. Lett. B478 (2000) 417.

[12] D. Besson et al. (CLEO Collaboration), Phys. Rev. D80 (2009) 032005.

[13] S. Aoki et al. (FLAG Working Group), arXiv:1607.00299 [hep-lat].

[14] B. Fahy and S. Hashimoto (JLQCD Collaboration), PoS (LATTICE2016) 118. 